\title{
Wall-less vascular poly(vinyl) alcohol gel ultrasound imaging phantoms using 3D printed vessels
}

Eleanor C. Mackle, Efthymios Maneas, Callum Little, Elizabeth Carr, Wenfeng Xia, et al.

Eleanor C. Mackle, Efthymios Maneas, Callum Little, Elizabeth Carr, Wenfeng Xia, Daniil Nikitichev, Roby D. Rakhit, Malcolm C. Finlay, Adrien E. Desjardins, "Wall-less vascular poly(vinyl) alcohol gel ultrasound imaging phantoms using 3D printed vessels ," Proc. SPIE 10870, Design and Quality for Biomedical Technologies XII, 108700P (27 February 2019); doi: 10.1117/12.2510033

SPIE. Event: SPIE BiOS, 2019, San Francisco, California, United States 


\title{
Wall-less vascular poly(vinyl) alcohol gel ultrasound imaging phantoms using 3D printed vessels
}

\author{
Eleanor C. Mackle*a,b Efthymios Maneas $^{\mathrm{a}, \mathrm{b}}$, Callum Little ${ }^{\mathrm{a}, \mathrm{b}, \mathrm{c}}$, Elizabeth Carr ${ }^{\mathrm{a}, \mathrm{b}}$, Wenfeng Xia ${ }^{\mathrm{a}, \mathrm{b}, \mathrm{d}}$ \\ Daniil Nikitichev $^{\mathrm{a}, \mathrm{b}}$, Roby D. Rakhit ${ }^{\mathrm{c}}$, Malcolm C. Finlay ${ }^{\mathrm{a}, \mathrm{e}}$, Adrien E. Desjardins ${ }^{\mathrm{a}, \mathrm{b}}$ \\ ${ }^{a}$ Department of Medical Physics and Bioengineering, University College London, Malet Place \\ Engineering Building, Gower Street, London WC1E 6BT, UK \\ ${ }^{\mathrm{b}}$ Wellcome-EPSRC Centre for Interventional \& Surgical Sciences \\ ${ }^{\mathrm{c}}$ Department of Cardiology, Royal Free Hospital, London \\ ${ }^{\mathrm{d}}$ School of Biomedical Engineering \& Imaging Sciences, King's College London, Lambeth Wing St. \\ Thomas' Hospital, Westminster Bridge Road, London SE1 7EH, UK \\ ${ }^{\mathrm{e}} \mathrm{Queen}$ Mary University of London and Barts Heart Centre
}

\begin{abstract}
Vascular phantoms are crucial tools for clinical training and for calibration and validation of medical imaging systems. With current methods, it can be challenging to replicate anatomically-realistic vasculature. Here, we present a novel method that enables the fabrication of complex vascular phantoms. Poly(vinyl alcohol) (PVA) in two forms was used to create wall-less vessels and the surrounding tissue mimicking material (TMM). For the latter, PVA cryogel (PVA-c) was used as the TMM, which was made from a solution of PVA (10\% by weight), distilled water, and glass spheres for ultrasonic scattering $(0.5 \%$ by weight). PVA-c is not water soluble, and after a freeze-thaw cycle it is mechanically robust. To form the wall-less vessels, vessel structures were 3D printed in water-soluble PVA and submerged in the aqueous solution of PVA-c. Once the PVA-c had solidified, the 3D printed PVA vessel structures were dissolved in water. Three phantoms were created, as initial demonstrations of the capabilities of this method: a straight vessel, a stenosed (narrowed), and a bifurcated (branched) vessel. Ultrasound images of the phantoms had realistic appearances. We conclude that this method is promising for creating wall-less, anatomically realistic, vascular phantoms.
\end{abstract}

Keywords: Phantoms, ultrasound, vessels, wall-less, poly(vinyl alcohol), 3D printing

*Correspondence to eleanor.mackle.14@ucl.ac.uk

\section{INTRODUCTION}

Vascular phantoms are important tools for clinical training, and for testing invasive medical devices and imaging systems. For instance, ultrasound-compatible vascular phantoms allow for Doppler studies of blood flow, which can be useful for peripheral vascular applications, where changes in flow can be indicative of pathology ${ }^{1}$. These phantoms typically comprise one or more vessels embedded in a tissue mimicking material (TMM) that has similar acoustic properties to those of soft biological tissues ${ }^{2}$. Ideally, vascular phantoms have mechanical and temporal stability, and are able to withstand physiological pressures and flow rates.

In previous vascular flow phantom studies, a wide range of TMM materials have been used, with varying levels of success. Agar ${ }^{3,4}$ and gelatin ${ }^{5,6}$ are often used as they are well established TMMs and simple to use; however, these materials do not have high mechanical stability or strength. Other materials such as silicone and gel wax have also been used $^{2}$. The review by Hoskins ${ }^{7}$ provides a useful overview of flow phantom materials and fabrication methods.

Vascular flow phantoms can be created with walled designs using solid vessel mimicking materials (VMMs), or with wall-less designs. Walled designs have been developed using hollow tubing, as reviewed by Law et al. ${ }^{8}$, but the small number of materials that have tissue-like ultrasonic properties and sufficient mechanical strength for phantom fabrication has been a limiting factor of this technique? ${ }^{9}$ Wall-less phantoms are sometimes preferred over walled phantoms, especially for smaller vessels ${ }^{10}$. Wall-less designs can reduce the acoustic mismatch between the TMM and the vessel

Design and Quality for Biomedical Technologies XII, edited by Rongguang Liang,

Jeeseong Hwang, T. Joshua Pfefer, and Gracie Vargas, Proc. of SPIE Vol. 10870,

108700P · @ 2019 SPIE · CCC code: 1605-7422/19/\$18 - doi: 10.1117/12.2510033 
wall, since the blood-mimicking fluid in the phantom is in direct contact with the TMM. There are many examples of wall-less vessel phantoms; fabrication methods include casting vessel structures with a low melting point metal that can be melted out of the $\mathrm{TMM}^{9,11}$, or using rods that can be extracted after the TMM has set, ${ }^{4,12}$. A prominent limitation of both walled and wall-less phantoms that is addressed here is that it can be challenging to replicate vessels with a high level of geometrical complexity. The phantom fabrication method described in this study, which involves 3D printed dissolvable PVA vessel structures, potentially allows for complex vascular anatomies to be realised.

In this study, water-insoluble poly(vinyl alcohol) cryogel (PVA-c) was used as a tissue mimicking material. PVA-c is attractive as a TMM: different properties can be tuned using the freeze-thaw cycles, it is mechanically robust, and it has an ultrasonic appearance similar to those of soft biological tissues ${ }^{13}$. Water-soluble, 3D-printed PVA, which is widely used as a printing support material ${ }^{14}$, was used to create wall-less vessels. The method presented here involves dissolving 3D printed vessel structures positioned within PVA-c, thereby creating wall-less vessels.

\section{METHODS AND MATERIALS}

\subsection{Vessel Design and Fabrication}

The various stages involved in the fabrication of a phantom using this technique are summarised in Fig. 1. Vessel structures for 3D printing were designed in Autodesk Fusion 360 (Autodesk, Inc., San Rafael, California, United States) and printed in PVA (RS Components, Corby, United Kingdom) on an Ultimaker 3 (Ultimaker, Geldermalsen, The Netherlands). These were closed structures, with $20 \%$ infill and a $1 \mathrm{~mm}$ thick shell. Three different designs were printed: a straight vessel, a stenosed (narrowed) vessel, and a bifurcated (branched) vessel. The 3D printed structures can be seen in Fig. 2. Vessels were all printed standing upright on the baseplate, with no support material used. The straight vessel was $120 \mathrm{~mm}$ in length, with a $10 \mathrm{~mm}$ outer diameter. The stenosed vessel was also $120 \mathrm{~mm}$ in length, with a $10 \mathrm{~mm}$ outer diameter, with a $50 \%$ stenosis in the middle section that was $30 \mathrm{~mm}$ long and $5 \mathrm{~mm}$ in diameter. The straight section of the bifurcated vessel was $70 \mathrm{~mm}$ long with a $10 \mathrm{~mm}$ outer diameter; each branch was $60 \mathrm{~mm}$ long. The two branches of the bifurcation had a $10 \mathrm{~mm}$ and $8 \mathrm{~mm}$ outer diameter, respectively, and the angle between them was $90^{\circ}$.

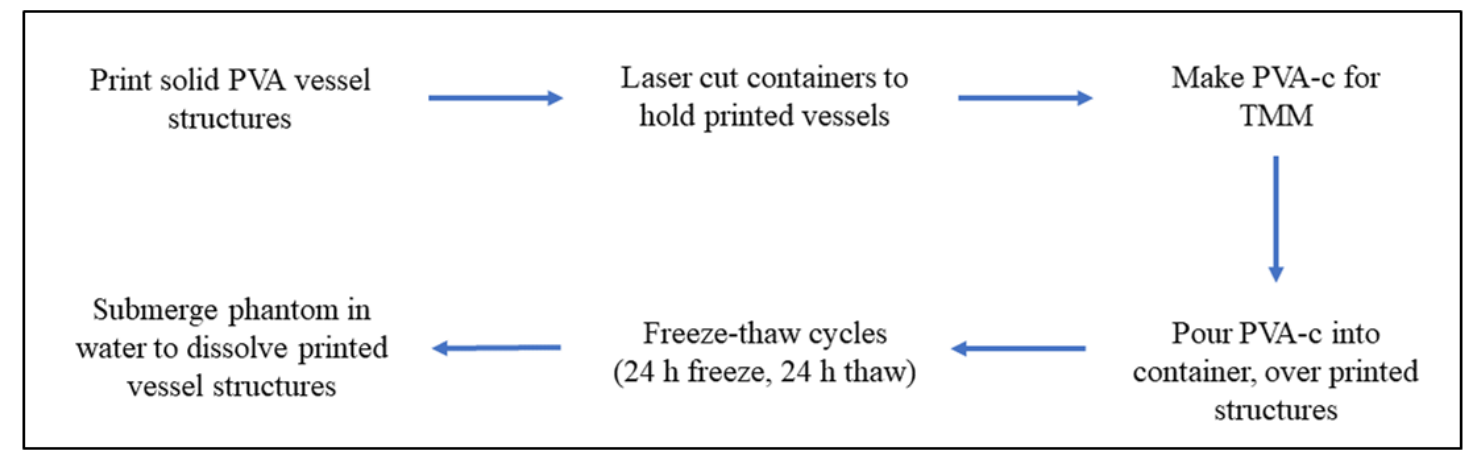

Figure 1. Primary phantom fabrication steps.

The PVA filament used for 3D printing can absorb water from the surrounding environment, which affects the quality of printed models; therefore, care was taken to ensure the PVA used was dry prior to use. When not in use, spools of PVA were kept in a sealed contained along with a desiccant. It was also found that the PVA printed well at $200{ }^{\circ} \mathrm{C}$, but above this temperature there was a tendency of the PVA to burn, so that the print quality was reduced and the printed vessels had exteriors that were less smooth. During printing, the baseplate was heated to $60{ }^{\circ} \mathrm{C}$, to provide good adhesion to the plate.

Preliminary studies showed that in some instances, the aqueous solution used to create PVA-c partially dissolved the printed structures before it has solidified. Therefore, printed structures were coated in parylene $(\sim 3 \mu \mathrm{m})$ to waterproof them and stop them from dissolving before intended. It was also found that hollow 3D printed PVA vessel structures dissolved much more quickly than solid ones. 

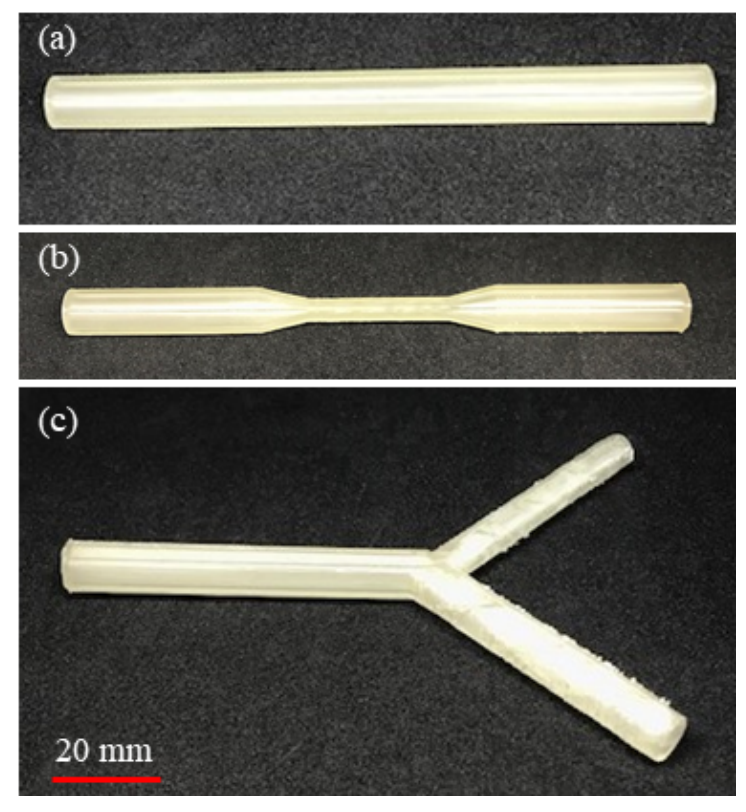

Figure 2. Photos of 3D printed vessel structures: (a) straight (b) stenosed (c) bifurcated. The scale bar applies to all photos.

\subsection{Containers}

Containers were made to shape and encapsulate the phantoms by laser cutting and combining sheets of acrylic. Holes in each end face of the container allowed printed vessel structures to be slotted in. These containers allowed the phantoms to be free standing and portable. For the straight and stenosed phantom, the box dimensions were $50 \mathrm{~mm} \times 50 \mathrm{~mm} \times 100$ $\mathrm{mm}$; for the bifurcation phantom, the box dimensions were $100 \mathrm{~mm} \times 50 \mathrm{~mm} \times 112 \mathrm{~mm}$.

\subsection{Printing Resolution Tests}

A test run was performed to investigate the minimum dimensions for 3D printed vessel structures. Vessel 'stumps' with small lengths $(30 \mathrm{~mm})$ were designed in Autodesk Fusion 360 and then printed in increasingly smaller sizes, until the prints failed. Both hollow and solid stumps were printed.

\subsection{Phantom Preparation}

The PVA-c used for the TMM was made with an aqueous solution of PVA powder and deionized water, using a method adapted from Kharine et $\mathrm{al}^{15}$. The solution was mixed in a temperature controlled water bath (HBR4 control, IKA), with an electronic stirrer (Eurostar Digital 20, IKA) and a 2 L conical flask.

The PVA-c was made in batches of $1500 \mathrm{ml}$, as this amount of solution was found to be optimal for the apparatus; if smaller batches were made, there was not enough solution in the conical flask for adequate and homogeneous stirring. The aqueous solution comprised 150 g PVA powder (Sigma Aldrich 99\%+ hydrolysed, average molecular weight $85,000-140,000)$ and $1350 \mathrm{ml}$ of deionized water. The deionized water was par boiled, then added to the conical flask. The flask was held within the water bath $\left(90^{\circ} \mathrm{C}\right)$, and the electronic stirrer was placed into the conical flask and set to $1800 \mathrm{rpm}$. When the water bath reached the set temperature, PVA powder was added gradually over $30 \mathrm{~min}$. The solution was then left to stir for $90 \mathrm{~min}$ to allow for the PVA powder to completely dissolve. Subsequently, the conical flask was removed from the water bath and the solution was left to cool at room temperature for $10 \mathrm{~min}$. The solution was then poured into the phantom containers with the $3 \mathrm{D}$ printed vessel structures positioned inside.

The phantoms were left to cool for an hour at room temperature before a freeze-thaw cycle was started. This freeze-thaw cycle involved freezing at $-20{ }^{\circ} \mathrm{C}$ for $24 \mathrm{~h}$ and thawing at $5{ }^{\circ} \mathrm{C}$ for $24 \mathrm{~h}$. Afterwards, the phantoms were submerged in deionized water for at least $48 \mathrm{~h}$ to dissolve out the printed structures. 


\subsection{Ultrasound Scanning}

Ultrasound images of the phantoms were acquired using a clinical scanner (MDP Ultrasonix, Richmond, Canada) and a linear array probe (14-5 MHz). Phantoms were submerged in water for imaging, to allow for coupling between the probe and the TMM and to remove air from the wall-less vessels.

\section{RESULTS}

\subsection{Printing Resolution Tests}

Solid 3D printed vessel structures ('stumps'), are shown in Fig. 3. Only vessels with a diameter greater than $5 \mathrm{~mm}$ printed smoothly; for those with smaller diameters, there tended to be excess material around the edges that was not on the original designs. These limitations notwithstanding, it was possible to print solid vessels down to diameters of $1 \mathrm{~mm}$. Vessels with a diameter less than $1 \mathrm{~mm}$ could not be printed, which was consistent with the print core, which had a 0.8 mm nozzle.
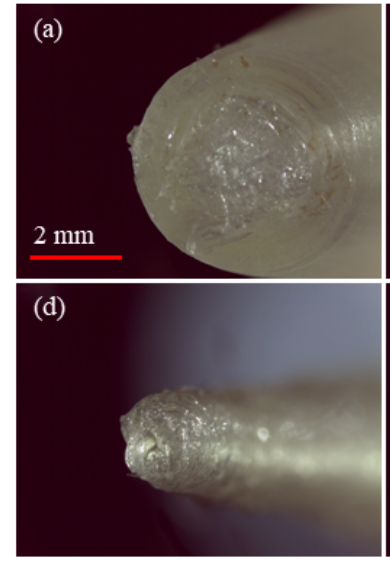

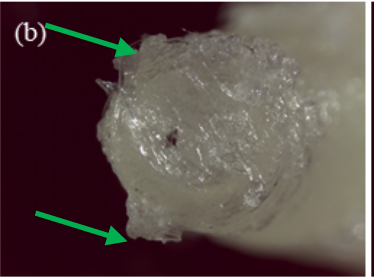

(e)

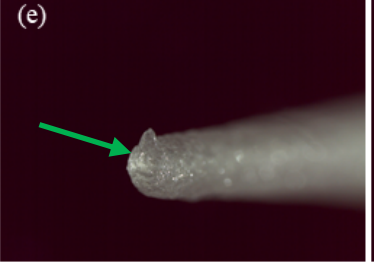

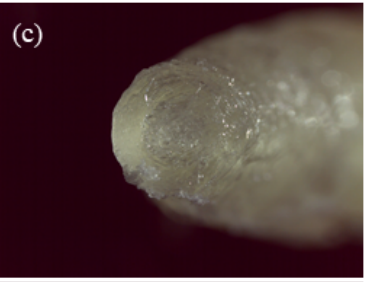

(f)

Figure 3. Solid 3D printed PVA vessel structures ('stumps') of decreasing design diameters, used to test 3D printing capabilities: (a) $5 \mathrm{~mm}$, (b) $4 \mathrm{~mm}$ (c) $3 \mathrm{~mm}$ (d) $2 \mathrm{~mm}$ (e) $1.5 \mathrm{~mm}$ (f) $1 \mathrm{~mm}$. The scale bar applies to each photo. Arrows in (b) and (e) indicate excess material that was not on the original designs.

Hollow 3D printed vascular structures ('stumps') with an outer diameter as small as $3 \mathrm{~mm}$ could be printed (Fig. 4). These structures tended to be much smoother than the solid ones, which may have resulted from differences in the way the print was built up. The smallest achievable thickness of the vessel wall was $1 \mathrm{~mm}$, using a print core with a $0.8 \mathrm{~mm}$ nozzle. Below $3 \mathrm{~mm}$, the vascular structures could be printed, but they were not hollow.

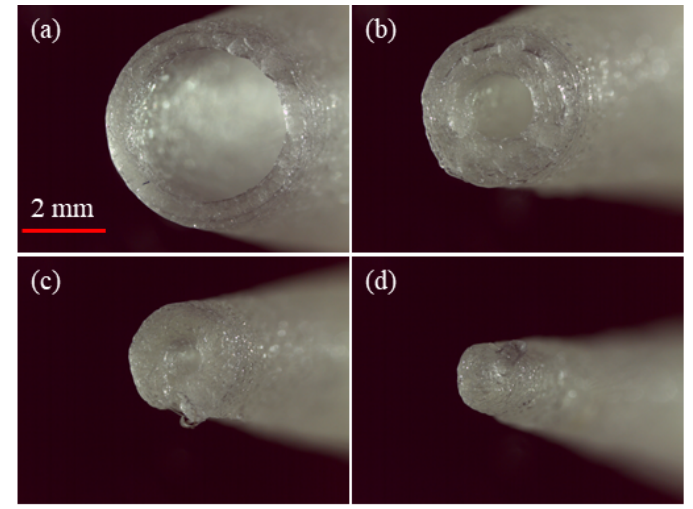

Figure 4. Hollow vessels of decreasing diameter ('stumps') and varying wall thickness used to test 3D printing capabilities: (a) $5 \mathrm{~mm}$ outer diameter, $4 \mathrm{~mm}$ inner diameter, (b) $4 \mathrm{~mm}$ outer diameter, $2 \mathrm{~mm}$ inner diameter (c) $3 \mathrm{~mm}$ outer diameter, 1.5 $\mathrm{mm}$ inner diameter (d) $2 \mathrm{~mm}$ outer diameter, $1 \mathrm{~mm}$ inner diameter. These values correspond to the designs and were not realised in all cases due to limitations of the $3 \mathrm{D}$ printing process. The scale bar applies to all images. 


\subsection{Phantoms}

A completed straight vessel phantom is shown in Fig. 5 (a). The opaque white appearance of the PVA-c that results from one freeze-thaw cycle was apparent. With ultrasound imaging, the wall-less vessel was seen to be patent with a smooth wall [Fig. 5(b)]. The hyperechoic region at the vessel wall may have resulted from an impedance mismatch between the TMM and the water in the vessel. The TMM had a tissue-like appearance; inhomogeneities may have resulted from incomplete mixing of the glass spheres. The stenosed phantom and a corresponding ultrasound image are shown in Fig. 6. In both Fig.5 (b) and Fig.6 (b) , the size of the vessel on ultrasound imaging is consistent with the designs.

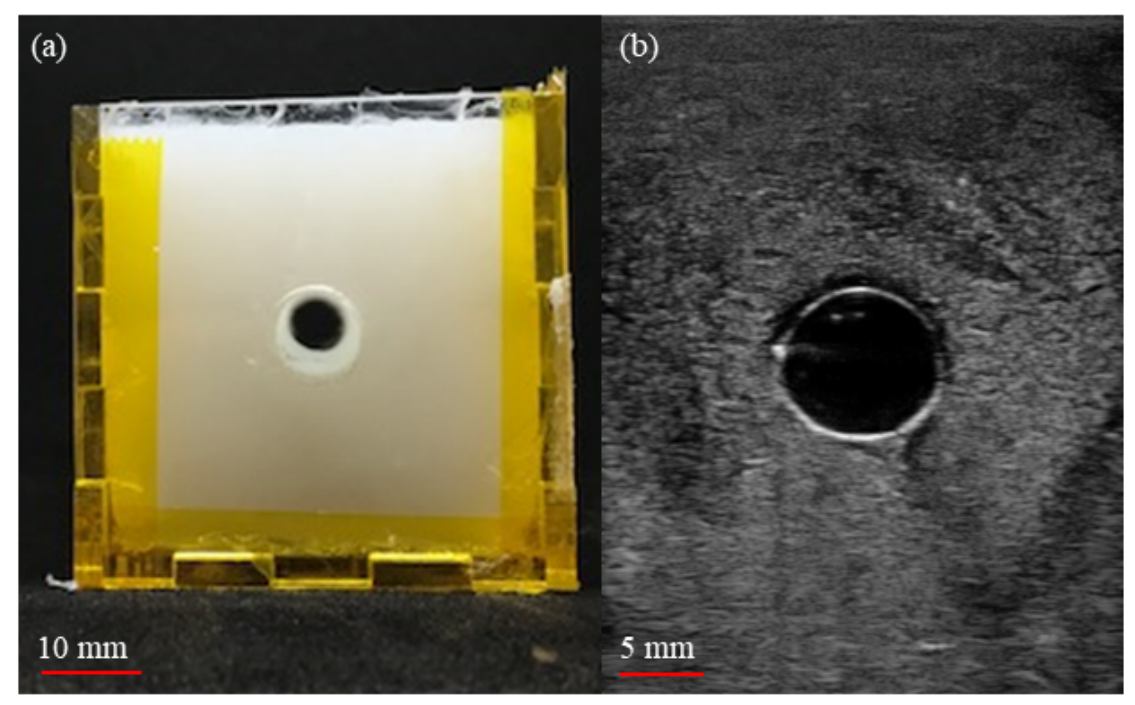

Figure 5. (a) Photo (side view) of the finished straight-vessel phantom, after one freeze-thaw cycle. Tape (yellow) was used to hold the acrylic sheets together in place of glue, for ease of fabrication and re-use. (b) Cross-sectional ultrasound image of the phantom.
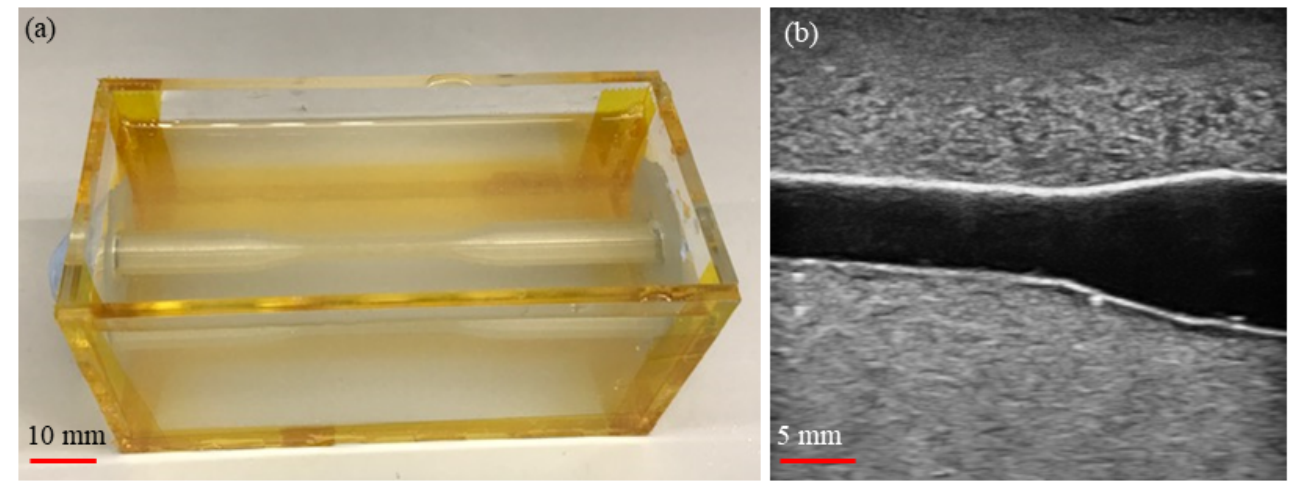

Figure 6. (a) Photo of the stenosis phantom (angled top view), with the aqueous solution of PVA-c added and before one freeze-thaw cycle. (b) Longitudinal ultrasound image of the vessel.

The bifurcation phantom is shown in Fig. 7 (a). The transparency of the aqueous solution used to create PVA-c, before a freeze-thaw cycle, is apparent. With ultrasound imaging, the two separate branches within the phantom were clearly visible; the left branch is noticeably narrower in diameter than the right branch, which was consistent with the design. Both vessel branches appear on the ultrasound image to be the same size as they were in the vessel designs, within 0.5 $\mathrm{mm}$. As with the straight-vessel phantom, there were inhomogeneities in the surrounding TMM. 

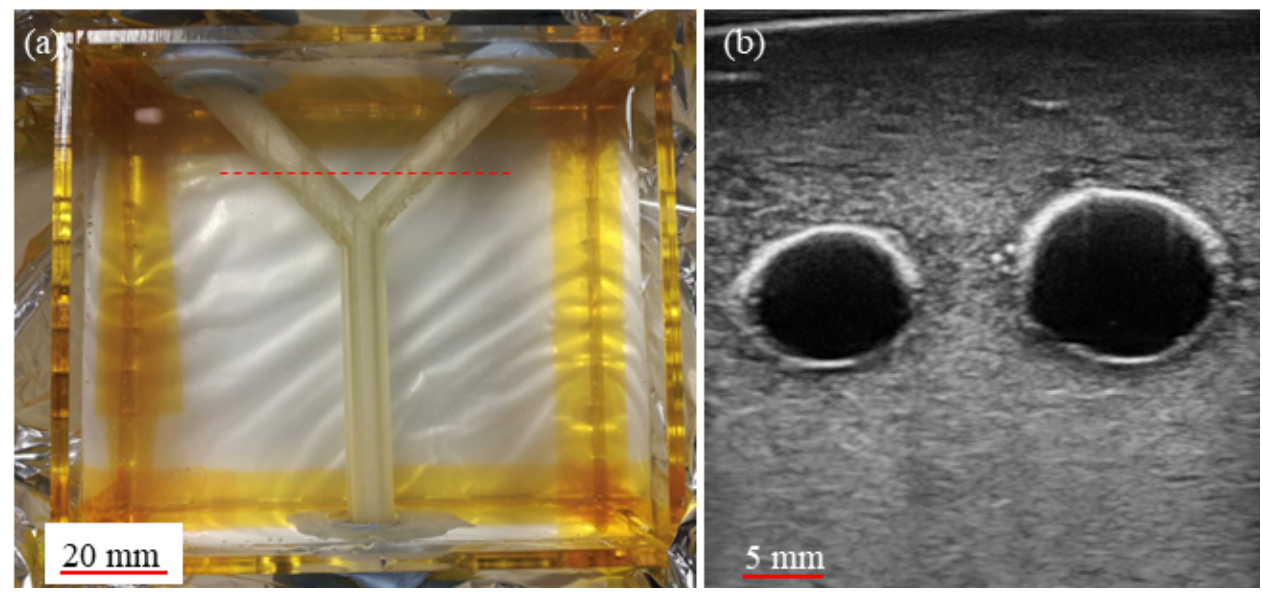

Figure 7. (a) Photo of the bifurcation phantom (top view) with the solution used to create PVA-c added and before a freezethaw cycle. (b) Cross-sectional ultrasound image of the phantom. The red dashed line in (a) indicates the ultrasound imaging plane.

\section{CONCLUSIONS}

Wall-less, ultrasound compatible vascular phantoms were successfully fabricated, using PVA in two different forms. 3D printing with water soluble PVA has the potential to overcome challenges of previous methods and to enable more complex geometries to be realised. Three basic geometries were demonstrated here: a straight vessel, a bifurcated vessel, and a stenosed vessel. These wall-less phantom geometries have been realised using rod based fabrication methods ${ }^{4}$. However, the key advantage of the novel method presented here is the potential to create more complex structures. The method described here could be improved with the use of support materials, and the use of smaller diameter print cores to enable printing of finer details and smoother surfaces. In this study, ultrasound imaging was the only modality used. In the future, adaptations involving constituents of the TMM and the blood mimicking fluid could allow for compatibility with other modalities such as MRI, CT, and photoacoustic imaging. We conclude that the combination of PVA-c and 3D printed PVA is a promising method for creating wall-less, anatomically realistic, vascular phantoms.

\section{REFERENCES}

[1] Cheng, C., Tempel, D., van Haperen, R., van der Baan, A., Grosveld, F., Daemen, M.J., Krams, R. and de Crom, R., "Atherosclerotic lesion size and vulnerability are determined by patterns of fluid shear stress." Circulation 113(23), 2744-2753 (2006).

[2] Maneas, E., Xia, W., Nikitichev, D.I., Daher, B., Manimaran, M., Wong, R.Y.J., Chang, C.W., Rahmani, B., Capelli, C., Schievano, S. and Burriesci, G., "Anatomically realistic ultrasound phantoms using gel wax with 3D printed moulds." Physics in Medicine \& Biology 63(1), 015033 (2018).

[3] Poepping, T.L., Nikolov, N., Rankin, N., Lee, M. and Holdsworth, D.W., "An in vitro system for Doppler ultrasound flow studies in the stenosed carotid artery bifurcation." Ultrasound in medicine \& biology 28(4), 495-506 (2002).

[4] Nikitichev, D.I., Barburas, A., McPherson, K., Mari, J.M., West, S.J. and Desjardins, A.E., "Construction of 3 dimensional printed ultrasound phantoms with wall less vessels." Journal of Ultrasound in Medicine 35(6), 1333-1339 (2016).

[5] Madsen, E.L., Hobson, M.A., Shi, H., Varghese, T. and Frank, G.R., "Tissue-mimicking agar/gelatin materials for use in heterogeneous elastography phantoms." Physics in Medicine \& Biology 50(23), 5597 (2005).

[6] Esmonde-White, F.W., Esmonde-White, K.A., Kole, M.R., Goldstein, S.A., Roessler, B.J. and Morris, M.D., "Biomedical tissue phantoms with controlled geometric and optical properties for Raman spectroscopy and tomography." Analyst 136(21), 4437-4446 (2011).

[7] Hoskins, P.R., "Simulation and validation of arterial ultrasound imaging and blood flow." Ultrasound in medicine \& biology 34(5), 693-717 (2008). 
[8] Law, Y.F., Johnston, K.W., Routh, H.F. and Cobbold, R.S.C., "On the design and evaluation of a steady flow model for Doppler ultrasound studies." Ultrasound in medicine \& biology 15(5), 505-516 (1989).

[9] Poepping, T.L., Nikolov, H.N., Thorne, M.L. and Holdsworth, D.W., "A thin-walled carotid vessel phantom for Doppler ultrasound flow studies." Ultrasound in medicine \& biology 30(8), 1067-1078 (2004).

[10] Rickey, D.W., Picot, P.A., Christopher, D.A. and Fenster, A., "A wall-less vessel phantom for Doppler ultrasound studies." Ultrasound in medicine \& biology 21(9), 1163-1176 (1995).

[11] King, D.M., Moran, C.M., McNamara, J.D., Fagan, A.J. and Browne, J.E., "Development of a vesselmimicking material for use in anatomically realistic Doppler flow phantoms." Ultrasound in medicine \& biology 37(5), 813-826 (2011).

[12] Ramnarine, K.V., Anderson, T. and Hoskins, P.R., "Construction and geometric stability of physiological flow rate wall-less stenosis phantoms." Ultrasound in medicine \& biology 27(2), 245-250 (2001).

[13] Fromageau, J., Brusseau, E., Vray, D., Gimenez, G. and Delachartre, P., "Characterization of PVA cryogel for intravascular ultrasound elasticity imaging." IEEE transactions on ultrasonics, ferroelectrics, and frequency control 50(10), 1318-1324 (2003).

[14] Kamran, M. and Saxena, A., "A comprehensive study on 3D printing technology." MIT Int J Mech Eng 6(2), 63-69 (2016).

[15] Kharine, A., Manohar, S., Seeton, R., Kolkman, R.G., Bolt, R.A., Steenbergen, W. and de Mul, F.F., "Poly (vinyl alcohol) gels for use as tissue phantoms in photoacoustic mammography." Physics in Medicine \& Biology 48(3), 357 (2003). 